\title{
Investigating Trk Protein Expression between Oropharyngeal and Non-oropharyngeal Squamous Cell Carcinoma: Clinical Implications and Possible Roles of Human Papillomavirus Infection
}

\author{
Yoon Ah Cho, MD \\ Ji Myung Chung, MS² \\ Hyunmi Ryu, PhD' \\ Eun Kyung Kim, MD, PhD ${ }^{1}$ \\ Byoung Chul Cho, MD, PhD 3 \\ Sun Och Yoon, MD, PhD ${ }^{1}$
}

${ }^{1}$ Department of Pathology, Severance Hospital, Yonsei University College of Medicine, Seoul, ${ }^{2}$ Yonsei University College of Medicine, Seoul, ${ }^{3}$ Division of Medical Oncology, Yonsei Cancer Center, Yonsei University College of Medicine, Seoul, Korea

\author{
Correspondence: Sun Och Yoon, MD, PhD \\ Department of Pathology, Severance Hospital, \\ Yonsei University College of Medicine, 50-1 \\ Yonsei-ro, Seodaemun-gu, Seoul 03722, Korea \\ Tel: 82-2-2228-1763 \\ Fax: 82-2-362-0860 \\ E-mail: soyoon@yuhs.ac
}

Received July 18, 2018

Accepted October 23, 2018

Published Online October 24, 2018

\begin{abstract}
Purpose
The relationship between head and neck squamous cell carcinoma (HNSCC) and subtypes of tropomyosin-related kinase (Trk) has not been studied in-depth. In this study, we evaluated the expression patterns of TrkA, TrkB, and panTrk and their clinicopathological significance as well as association with p16 expression and human papillomavirus (HPV) status.
\end{abstract}

\section{Materials and Methods}

Total of 396 radically resected oropharyngeal $(n=121)$ and non-oropharyngeal $(n=275)$ HNSCCs were included. Immunohistochemistry for TrkA, TrkB, and panTrk was performed. In addition, p16 immunohistochemistry was performed to assess the HPV status. Using HPV-negative HNSCC cell lines, FaDu and CAL27, HPV type 16 E6/E7 gene was transfected, and then changes of TrkA and TrkB expression were analyzed.

\section{Results}

In the clinical samples of HNSCC, high expression of TrkA and panTrk were more associated with oropharyngeal and $\mathrm{p} 16$ positive squamous cell carcinoma (SCC). In patients with completely resected (RO-resected) oropharyngeal SCC, high TrkA expression was related to superior overall survival and recurrence-free survival (RFS). In patients with R0-resected oral cavity SCC, high panTrk was related to poor RFS. In HPV type E6/E7 gene-transfected FaDu and CAL27 cell lines, increase of TrkA expression was observed.

\section{Conclusion}

It seems that expression pattern of panTrk and TrkA differed according to anatomical sites of HNSCC and was closely related to $\mathrm{p} 16$ expression and patient prognosis. Trk expression should be considered in the context of anatomical site, p16 expression or HPV status and Trk subtypes.

\section{Introduction}

Head and neck squamous cell carcinoma (HNSCC) is a rare cancer [1]. Recurrence and metastasis occur in about one third of all HNSCC patients. When recurrence or metastasis occurs, the outcomes are dismal [2]. Therefore, it is desirable to have alternative treatment options for such cases.

Oropharyngeal cancer, in contrast to other non-oropharyngeal head and neck cancers, is known to be related to human
Key words

Head and neck squamous cell carcinoma, Trk proteins, TrkA, TrkB, panTrk, Human papillomavirus papilloma virus (HPV) infection, and patients with HPV-positive oropharyngeal cancer have a better prognosis than those with HPV-negative cancer [3]. According to the recent World Health Organization (WHO) classification [4,5], oropharyngeal squamous cell carcinoma (SCC) is classified as HPV-positive and HPV-negative oropharyngeal SCC. Regarding 'HPV-positive' and 'HPV-negative,' the new WHO classification $[4,5]$ specifically note p16 immunohistochemistry alone (with appropriate criteria for a positive versus negative test) as a suitable surrogate marker and recommend the ter- 
minology HPV-positive even if only p16 is performed. Using moderate to intense nuclear and cytoplasmic p16 immunohistochemistry in $70 \%$ or more of the tumor cells as surrogate marker for HPV status has been widely validated, especially in oropharyngeal SCC [6,7]. HPV status appears to affect tumor characteristics in patients with oropharyngeal cancer, but the underlying mechanism has not yet been clarified.

Trophomyosin-related kinases (Trk), namely TrkA, TrkB, and TrkC, belong to a family of neurotrophin receptor tyrosine kinases and are encoded by the NTRK1, NTRK2, and NTRK3 genes, respectively. TrkA, TrkB, and TrkC bind to nerve growth factor, brain-derived neurotrophic factor, and neurotrophin 3, respectively [8]. In HNSCC, TrkB is known to be overexpressed and related to oncogenesis, tumor progression, and conventional chemotherapy resistance $[9,10]$.

While attempts to describe Trk expression patterns have drawn increasing interest, Trk expression status has rarely been studied in clinical samples of HNSCC. In this study, we sought to evaluate the expression status of Trk in oropharyngeal cancer and non-oropharyngeal cancer and to determine its clinicopathological significance. In addition, possible associations between HPV status, p16 expression and Trk protein expression were investigated.

\section{Materials and Methods}

\section{Patients, samples, and clinical data}

Formalin-fixed, paraffin-embedded (FFPE) specimens were obtained from consecutive HNSCC patients who underwent surgical resection with curative aim at Severance Hospital, Seoul, Korea, between 2005 and 2012. Appropriate cases were selected from among the archived cases. Inclusion criteria were as follows: available tumor tissue, relevant clinical data regarding smoking status and survival data, absence of preoperative treatment, and no clinicopathologic evidence of distant metastasis at the time of surgery. We excluded HNSCC tissue samples that had been subjected to decalcification for accurate immunohistochemistry. Ultimately, 121 oropharyngeal cases and 275 non-oropharyngeal cases, total 396 cases were selected, among which complete R0 resection,
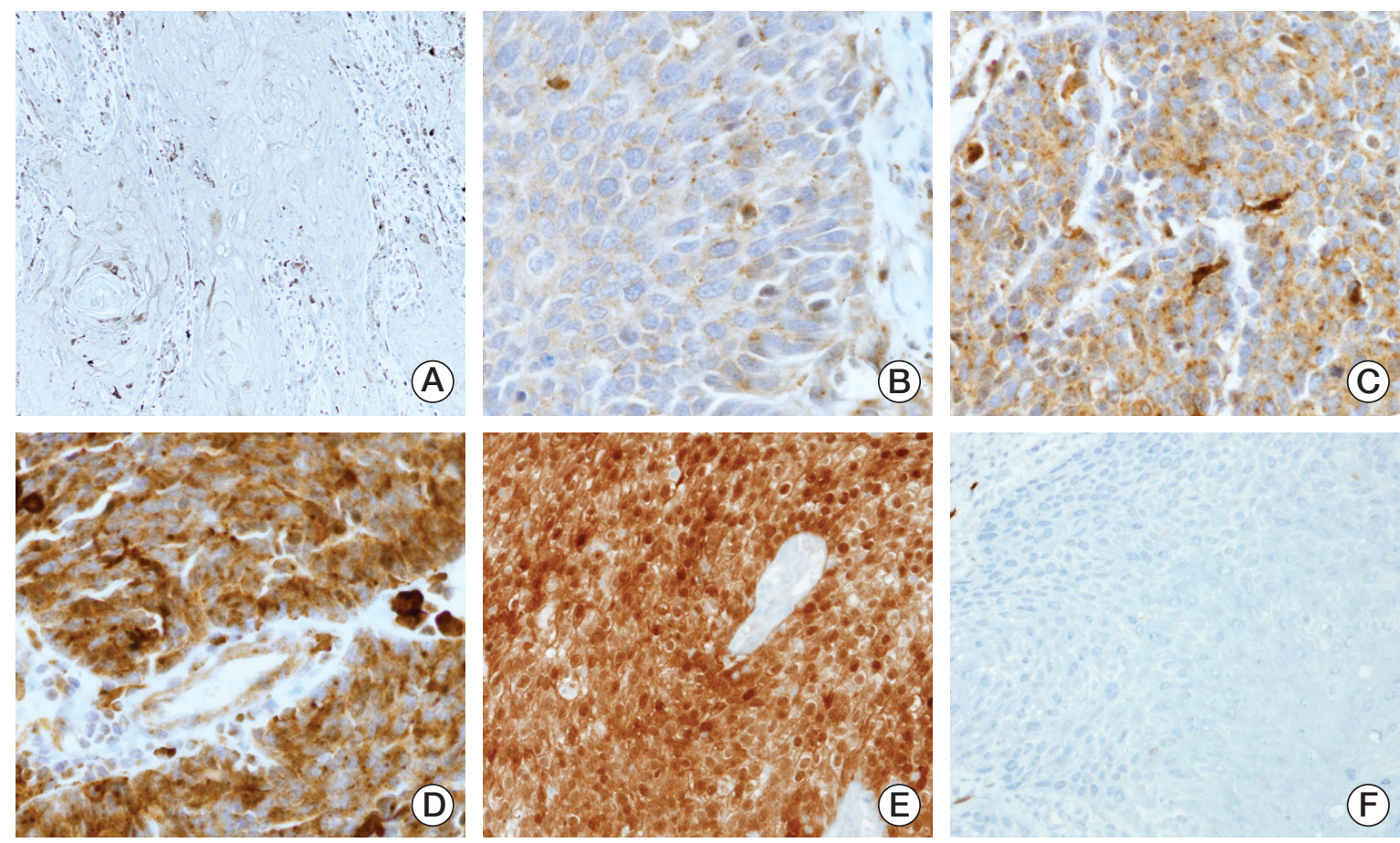

Fig. 1. Expression pattern of Trk protein and p16 using immunohistochemistry $(\times 200)$. Representative expression as assessed by Trk immunohistochemistry: Trk showed diffuse cytoplasmic staining in tumor cells. The four cases shown had different intensities of Trk expression in all tumor cells. (A) Negative, H-score 0. (B) Intensity 1, H-score 100. (C) Intensity 2, H-score 200. (D) Intensity 3, H-score 300. Strong and diffuse nuclear and cytoplasmic staining of p16 immunohistochemical staining (E) and negative staining was observed (F). 
defined histologically as tumor-free resection margins, achieved in 305 (S1 Table). Several pathologic factors, including tumor size, lymphovascular invasion, perineural invasion, pathologic TNM staging, according to the 7th American Joint Committee on Cancer (AJCC) criteria and tumor classification by the WHO system $[11,12]$, were obtained from the slide review by two individual pathologists (Y.A. Cho and S.O. Yoon). Clinical data and survival outcomes were collected and reviewed from patients' medical records. The median follow-up period was 37.1 months (range, 0.8 to 99.6 months). Other clinicopathologic characteristics are described in S1 Table.

\section{Tissue microarray preparation}

Sections of FFPE tissues were prepared and stained with hematoxylin and eosin. Representative tumor areas were confirmed microscopically, selected two or three different representative areas per case, and used for tissue microarray (TMA) construction as previously described [13].

\section{Immunohistochemistry and interpretation}

Immunohistochemistry was performed on 4- $\mu$ m TMA tissue sections with a Ventana Bench Mark XT Autostainer (Ventana Medical Systems, Tucson, AZ) as described in previous reports $[14,15]$. The following primary antibodies were tested: anti-TrkA antibody (1:100 dilution, EP1058Y, rabbit monoclonal antibody, Abcam, Cambridge, MA), anti-TrkB antibody (1:300 dilution, ab18987, rabbit polyclonal antibody, Abcam), anti-panTrk (1:100 dilution, Trk A+B+C, EPR17341, rabbit monoclonal antibody, Abcam), and p16 (E03347, mouse monoclonal antibody, RTU, Ventana Medical Systems).

Expression of Trk subtypes was analyzed according to the semi-quantitative $\mathrm{H}$-score method; this method yields a total score range of 0-300 as previously described [16]. The intensity of expression was scored by cytoplasmic staining only (Fig. 1A-D). Conventionally accepted criteria were used for p16 immunohistochemistry as previously described. Positive and negative pattern is described in Fig. 1E and F respectively [17-22].

\section{Cell lines and culture}

HNSCC cell lines FaDu, a cell line derived from HPV-negative pharynx SCC, and CAL27, a cell line derived from HPV-negative tongue SCC were prepared. FaDu cell line was maintained in RPMI-1640 medium (22400-089, Gibco, Life Technologies, Carlsbad, CA) supplemented with $10 \%$ fetal bovine serum (FBS; 5000-044, Gibco, Life Technologies). CAL27 and UMSCC 47 cell lines were maintained in Dulbecco's modified Eagle's medium (SH30243.01, HyClone,
Thermo Scientific, Logan, UT) supplemented with 10\% FBS (6000-044, Gibco, Life Technologies).

\section{Transfection with HPV gene}

Plasmid p1321 HPV-16 E6/E7 or empty plasmid vector (Addgene, Cambridge, MA) was transfected into each cellline of FaDu and CAL27 by using TransIT-X2 Dynamic Delivery System (Mirus, Madison, WI).

\section{RNA isolation and real-time polymerase chain reaction}

RNA was isolated after 24 hours and 48 hours of transfection using RNeasy plus mini kit (Qiagen, Valencia, CA). cDNA was made using qPCRBIO cDNA synthesis kit (PCR Biosystems, London, UK). Primers of GAPDH, E6, E7, TrkA, TrkB, and TrkC were manufactured (Integrated DNA Technologies, Skokie, IL), and primer sequences were summarized in S2 Table. Quantitative polymerase chain reaction (PCR) was performed using $2 \times$ qPCRBIO SyGreen Mix Lo-Rox (PCR Biosystems) as PCR reagent and ABI 7500 RealTime PCR System (Applied Biosystems, Thermo Fisher Scientific) with the following cycling parameters: $95^{\circ} \mathrm{C}$ for 2 minutes, 40 cycles of $95^{\circ} \mathrm{C}$ for 5 seconds and $60^{\circ} \mathrm{C}$ for 30 seconds, $95^{\circ} \mathrm{C}$ for 15 seconds, and $60^{\circ} \mathrm{C}$ for 10 minutes. Relative mRNA expression levels were determined by the comparative method ( $\left.2^{\text {-ddCt }}\right)$.

\section{Statistical analysis}

Statistical analyses were conducted using IBM SPSS ver. 22 software for Windows (IBM Corp., Armonk, NY). The Mann-Whitney U test, Kruskal-Wallis test and chi-square test, were used to analyze the significance of differences between the evaluated variables. Overall survival (OS) was measured from the date of initial diagnosis to that of death or the last follow-up visit. Recurrence-free survival (RFS) was measured from the date of initial diagnosis to that of disease progression, defined as cancer recurrence, continuance of stable disease/partial remission/progressive disease without complete remission, or cancer-related death during the study period. The Kaplan-Meier method was used to analyze survival rates, and differences were compared using the logrank test. Multivariate regression analysis was performed using a Cox proportional hazards model. Two-sided p-values $<0.05$ were considered statistically significant.

\section{Ethical statement}

This study was approved by the Institutional Review Board of Severance Hospital with a waiver of informed consent (No. 4-2015-0954). 

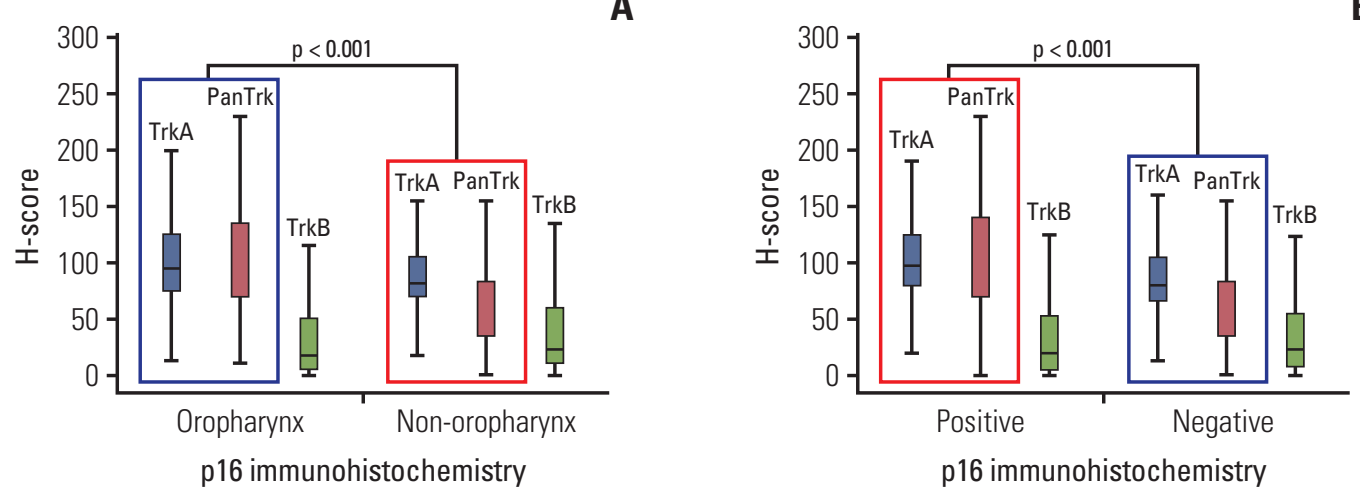

Fig. 2. Expression of Trk protein in head and neck squamous cell carcinomas according to anatomical location and p16 status. (A) H-scores of TrkA and panTrk were significantly higher at oropharyngeal sites than non-oropharyngeal areas $(\mathrm{p}<0.001)$. However, there was no significant difference in $\mathrm{H}$-scores of TrkB according to anatomical site. (B) H-scores of TrkA and panTrk were higher in p16 positive cases than p16 negative cases. No significant difference was observed in the $\mathrm{H}$-score of TrkB between p16 positive cases and p16 negative cases.

A

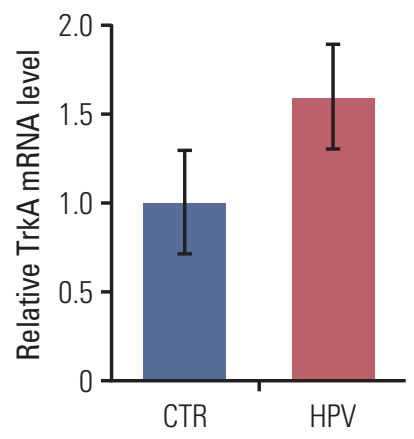

D

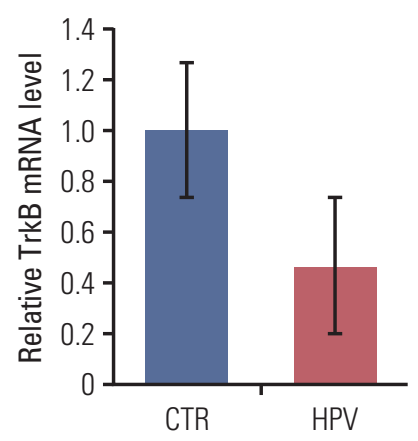

B

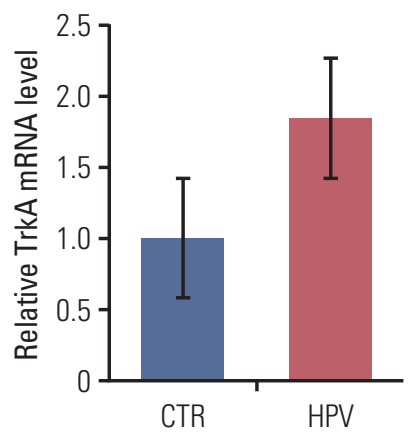

E

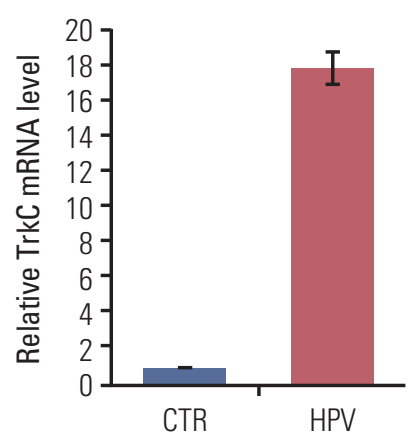

C

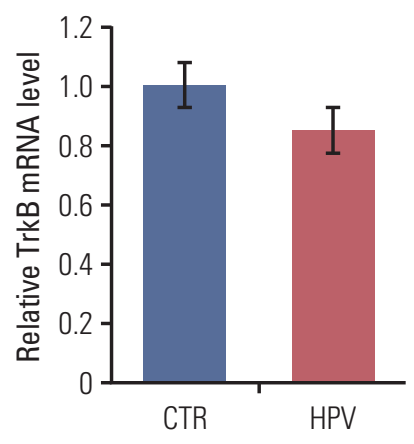

$\mathbf{F}$

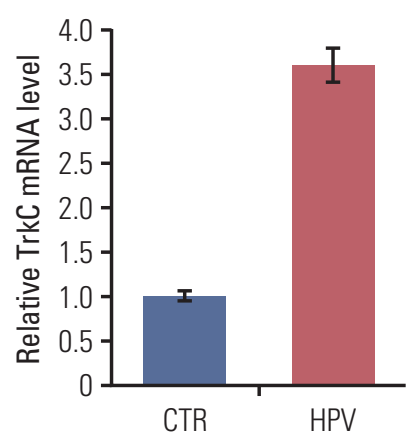

Fig. 3. Alteration of TrkA and TrkB expression and proliferation ability by HPV-16 oncogene. (A) Increased level of TrkA mRNA 48 hours after transfection in FaDu cell line. (B) Increased level of TrkA mRNA 48 hours after transfection in CAL27 cell-line. (C) Slight decreased level of TrkB mRNA in FaDu cell line. (D) Decreased level of TrkB in CAL27 cell line. Increased level of TrkC mRNA 48 hours after transfection in FaDu cell line (E) and CAL27 cell line (F). CTR, transfection with empty vector; HPV, transfection with HPV-16 oncogene. 
Table 1. Clinicopathological characteristics of 391 HNSCCs according to TrkA and panTrk expression status

\begin{tabular}{|c|c|c|c|c|c|c|c|}
\hline \multirow{2}{*}{ Variable } & \multirow{2}{*}{$\begin{array}{c}\text { No. } \\
\left.(\mathrm{n}=391)^{\mathrm{a}}\right)\end{array}$} & \multicolumn{2}{|c|}{ TrkA expression } & \multirow{2}{*}{ p-value } & \multicolumn{2}{|c|}{ panTrk expression } & \multirow{2}{*}{ p-value } \\
\hline & & High $(n=160)$ & Low $(n=231)$ & & High $(n=169)$ & Low $(n=222)$ & \\
\hline \multicolumn{8}{|l|}{ Age (yr) } \\
\hline$<58$ & 190 & $76(47.5)$ & $114(49.4)$ & 0.758 & $73(43.2)$ & $117(52.7)$ & 0.067 \\
\hline$\geq 58$ & 201 & $84(52.5)$ & $117(50.6)$ & & $96(56.8)$ & $105(47.3)$ & \\
\hline \multicolumn{8}{|l|}{ Sex } \\
\hline Female & 97 & $40(25.0)$ & $57(24.7)$ & $>0.999$ & $34(20.1)$ & $63(28.4)$ & 0.076 \\
\hline Male & 294 & $120(75.0)$ & $175(75.3)$ & & $135(79.9)$ & $159(71.6)$ & \\
\hline \multicolumn{8}{|l|}{ Location } \\
\hline Hypopharynx & 28 & $8(5.0)$ & $20(8.7)$ & 0.011 & $13(7.7)$ & $15(6.8)$ & $<0.001$ \\
\hline Larynx & 42 & $12(7.5)$ & $30(13.0)$ & & $16(9.5)$ & $26(11.7)$ & \\
\hline Oral cavity & 200 & $77(48.1)$ & $123(53.2)$ & & $57(33.7)$ & $143(64.4)$ & \\
\hline Oropharynx & 121 & $63(39.4)$ & $58(25.1)$ & & $83(49.1)$ & $38(17.1)$ & \\
\hline \multicolumn{8}{|l|}{ Smoking } \\
\hline Non-smoker & 151 & $70(43.8)$ & $81(35.1)$ & 0.214 & $55(32.5)$ & $96(43.2)$ & 0.085 \\
\hline Ex-smoker & 80 & $29(18.1)$ & $51(22.1)$ & & $40(23.7)$ & $40(18.0)$ & \\
\hline Smoker & 160 & $61(38.1)$ & $99(42.9)$ & & $74(43.8)$ & $86(38.7)$ & \\
\hline \multicolumn{8}{|l|}{ p16 IHC status } \\
\hline Negative & 269 & $88(55.0)$ & $181(78.4)$ & $<0.001$ & $86(50.9)$ & $183(82.4)$ & $<0.001$ \\
\hline Positive & 122 & $72(45.0)$ & $50(21.6)$ & & $83(49.1)$ & 39 (17.6) & \\
\hline \multicolumn{8}{|c|}{ Lymphovascular invasion } \\
\hline Absent & 315 & $118(73.8)$ & $197(85.3)$ & 0.004 & $119(70.4)$ & $196(88.3)$ & $<0.001$ \\
\hline Present & 76 & $42(26.3)$ & $34(14.7)$ & & $50(29.6)$ & $26(11.7)$ & \\
\hline \multicolumn{8}{|c|}{ Perineural invasion } \\
\hline Absent & 337 & $132(82.5)$ & $205(88.7)$ & 0.054 & $139(82.2)$ & $198(89.2)$ & 0.055 \\
\hline Present & 54 & $28(17.5)$ & $26(11.3)$ & & $30(17.8)$ & $24(10.8)$ & \\
\hline \multicolumn{8}{|c|}{ Pathologic T category } \\
\hline pT1-2 & 321 & $128(80.0)$ & $193(83.5)$ & 0.421 & $133(78.7)$ & $188(84.7)$ & 0.143 \\
\hline pT3-4 & 70 & $32(20.0)$ & $38(16.5)$ & & $36(21.3)$ & $34(15.3)$ & \\
\hline \multicolumn{8}{|c|}{ Lymph node metastasis } \\
\hline Absent & 172 & $62(38.8)$ & $110(47.6)$ & 0.097 & $53(31.4)$ & $119(53.6)$ & $<0.001$ \\
\hline Present & 219 & $98(61.3)$ & $121(52.4)$ & & $116(68.6)$ & $103(46.4)$ & \\
\hline \multicolumn{8}{|c|}{ Perinodal soft tissue extension ${ }^{\text {b) }}$} \\
\hline Absent & 52 & $25(34.7)$ & $27(35.1)$ & $>0.999$ & $24(29.6)$ & $28(41.2)$ & 0.169 \\
\hline Present & 97 & $47(65.3)$ & $50(64.9)$ & & $57(70.4)$ & $40(58.8)$ & \\
\hline
\end{tabular}

Values are presented as number (\%). HNSCC, head and neck squamous cell carcinoma; IHC, immunohistochemistry. ${ }^{\text {a }}$ TrkA and PanTrk immunohistochemistry was performed in all 396 cases but five cases could not be interpreted because of a dam-

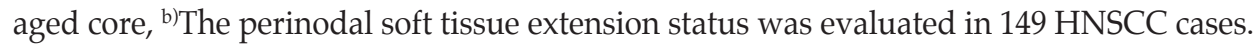

\section{Results}

\section{Expression status of Trk protein according to anatomical site and p16 expression}

In overall HNSCC cases, TrkA expression was on average 91.8 (range, 0 to 230), TrkB 36.8 (range, 0 to 278), and panTrk 79.2 (range, 0 to 263) among all HNSCC cases (S3 Fig.). Trk expression varied among anatomical sites. Expression of
TrkA and panTrk was significantly higher in oropharyngeal SCCs than non-oropharyngeal SCCs. Also both expressions were significantly higher in 16 positive HNSCCs than negative cases. However, TrkB expression showed no correlation with anatomical sites and p16 status (Fig. 2A and B, S4 and S5 Figs.). 
Table 2. Clinicopathological characteristics of 121 oropharyngeal HNSCCs according to TrkA and panTrk expression status

\begin{tabular}{|c|c|c|c|c|c|c|c|}
\hline \multirow{2}{*}{ Variable } & \multirow{2}{*}{$\begin{array}{c}\text { No. } \\
(n=121)\end{array}$} & \multicolumn{2}{|c|}{ TrkA expression } & \multirow{2}{*}{ p-value } & \multicolumn{2}{|c|}{ panTrk expression } & \multirow{2}{*}{ p-value } \\
\hline & & High $(n=63)$ & Low $(n=58)$ & & High $(n=83)$ & Low $(n=38)$ & \\
\hline \multicolumn{8}{|l|}{ Age (yr) } \\
\hline$<58$ & 57 & $27(42.9)$ & $30(51.7)$ & 0.365 & $38(45.8)$ & $19(50.0)$ & 0.698 \\
\hline$\geq 58$ & 64 & $36(57.1)$ & $28(48.3)$ & & $45(54.2)$ & $19(50.0)$ & \\
\hline \multicolumn{8}{|l|}{ Sex } \\
\hline Female & 20 & $8(12.7)$ & $12(20.7)$ & 0.328 & $14(16.9)$ & $6(15.8)$ & $>0.999$ \\
\hline Male & 101 & $55(87.3)$ & $46(79.3)$ & & $69(83.1)$ & $32(84.2)$ & \\
\hline \multicolumn{8}{|l|}{ Smoking } \\
\hline Non-smoker & 38 & $22(34.9)$ & $16(27.6)$ & 0.676 & $26(31.3)$ & 12 (31.6) & 0.345 \\
\hline Ex-smoker & 25 & $12(19.0)$ & $13(22.4)$ & & $20(24.1)$ & $5(13.2)$ & \\
\hline Smoker & 58 & $29(46.0)$ & $29(50.0)$ & & $37(44.6)$ & $21(55.2)$ & \\
\hline \multicolumn{8}{|l|}{ p16 IHC status } \\
\hline Negative & 29 & $6(9.5)$ & $23(39.7)$ & $<0.001$ & $15(18.1)$ & $14(36.8)$ & 0.038 \\
\hline Positive & 92 & $57(90.5)$ & $35(60.3)$ & & $68(81.9)$ & $24(63.2)$ & \\
\hline \multicolumn{8}{|c|}{ Lymphovascular invasion } \\
\hline Absent & 81 & $39(61.9)$ & $42(72.4)$ & 0.250 & $54(65.1)$ & $27(71.1)$ & 0.541 \\
\hline Present & 40 & $24(38.1)$ & $16(27.6)$ & & $29(34.9)$ & $11(28.9)$ & \\
\hline \multicolumn{8}{|c|}{ Perineural invasion } \\
\hline Absent & 111 & $56(88.9)$ & $55(94.8)$ & 0.327 & $75(90.4)$ & $36(94.7)$ & 0.503 \\
\hline Present & 10 & $7(11.1)$ & $3(5.2)$ & & $8(9.6)$ & $2(5.3)$ & \\
\hline \multicolumn{8}{|c|}{ Pathologic $\mathrm{T}$ category } \\
\hline pT1-2 & 101 & $50(79.4)$ & $51(87.9)$ & 0.230 & $69(83.1)$ & $32(84.2)$ & $>0.999$ \\
\hline pT3-4 & 20 & $13(20.6)$ & $7(12.1)$ & & $14(16.9)$ & $6(15.8)$ & \\
\hline \multicolumn{8}{|c|}{ Lymph node metastasis } \\
\hline Absent & 23 & $12(19.0)$ & $11(19.0)$ & $>0.999$ & $18(21.7)$ & $5(13.2)$ & 0.325 \\
\hline Present & 98 & $51(81.0)$ & $47(81.0)$ & & $65(78.3)$ & $33(86.8)$ & \\
\hline \multicolumn{8}{|c|}{ Perinodal soft tissue extension ${ }^{\text {a) }}$} \\
\hline Absent & 22 & $13(32.5)$ & $9(31.0)$ & $>0.999$ & $13(27.7)$ & $9(40.9)$ & 0.284 \\
\hline Present & 47 & $27(67.5)$ & $20(69.0)$ & & 34 (72.3) & $13(59.1)$ & \\
\hline
\end{tabular}

Values are presented as number (\%). HNSCC, head and neck squamous cell carcinoma; IHC, immunohistochemistry. ${ }^{\text {a) }}$ The perinodal soft tissue extension status was evaluated in 69 oropharyngeal HNSCC cases.

\section{Alteration of Trk protein expression by HPV gene trans- fection}

After transfection of HPV-16 E6 / E7 gene inserted plasmid, significant increase of E6 and E7 gene expression was confirmed at the time of 24 and 48 hours after transfection (S6 Fig.). The levels of TrkA, TrkB and TrkC mRNA level were measured 48 hours after transfection; and both FaDu and CAL27 transfected with HPV-16 E6/E7 genes revealed increased expression of TrkA and TrkC when compared to control (Fig. 3A, B, E and F). TrkB gene expression showed a tendency of decreased expression after HPV-16 E6/E7 gene transfection (Fig. 3C and D).

\section{Clinicopathological characteristics in conjunction with Trk expression}

Among all HNSCC cases, high TrkA and panTrk expression was significantly associated with the presence of lymphovascular invasion and p16 expression (Table 1). In addition, high panTrk expression was significantly associated with the presence of lymph node metastasis $(p<0.001)$. However, other clinicopathologic variables were not related to the expression of TrkA and panTrk proteins (Table 1). In oropharyngeal HNSCCs, only p16 expression was significantly associated with TrkA and panTrk protein expression ( $p<0.001$ and $p=0.038$, respectively) (Table 2). However, panTrk expression was significantly correlated with the presence of lymphovascular invasion ( $\mathrm{p}<0.001)$, presence of perineural invasion $(\mathrm{p}=0.007)$, advanced pathologic T category 
Table 3. Clinicopathological characteristics of 270 non-oropharyngeal HNSCCs according to Trk expression status

\begin{tabular}{|c|c|c|c|c|c|c|c|}
\hline \multirow{2}{*}{ Variable } & \multirow{2}{*}{$\begin{array}{c}\text { No. } \\
\left.(n=270)^{a}\right)\end{array}$} & \multicolumn{2}{|c|}{ TrkA expression } & \multirow{2}{*}{ p-value } & \multicolumn{2}{|c|}{ panTrk expression } & \multirow{2}{*}{ p-value } \\
\hline & & High $(n=97)$ & Low $(n=173)$ & & High $(n=86)$ & Low $(n=184)$ & \\
\hline \multicolumn{8}{|l|}{ Age (yr) } \\
\hline$<58$ & 133 & $49(50.5)$ & $84(48.6)$ & 0.800 & $35(40.7)$ & $98(53.3)$ & 0.067 \\
\hline$\geq 58$ & 137 & $48(49.5)$ & $89(51.4)$ & & $51(59.3)$ & $86(46.7)$ & \\
\hline \multicolumn{8}{|l|}{ Sex } \\
\hline Female & 77 & $32(33.0)$ & $45(26.0)$ & 0.261 & $20(23.3)$ & $57(31.0)$ & 0.247 \\
\hline Male & 193 & $65(67.0)$ & $128(74.0)$ & & $66(76.7)$ & $127(69.0)$ & \\
\hline \multicolumn{8}{|l|}{ Smoking } \\
\hline Non-smoker & 113 & $48(49.5)$ & $65(37.6)$ & 0.163 & $29(33.7)$ & $84(45.7)$ & 0.180 \\
\hline Ex-smoker & 55 & $17(17.5)$ & $38(22.0)$ & & $20(23.3)$ & $35(19.0)$ & \\
\hline Smoker & 102 & $32(33.0)$ & $70(40.5)$ & & $37(43.0)$ & $65(35.3)$ & \\
\hline \multicolumn{8}{|l|}{ p16 IHC status } \\
\hline Negative & 240 & $82(84.5)$ & $158(91.3)$ & 0.107 & $71(82.6)$ & $169(91.8)$ & 0.036 \\
\hline Positive & 30 & 15 (15.5) & $15(8.7)$ & & $15(17.4)$ & $15(8.2)$ & \\
\hline \multicolumn{8}{|c|}{ Lymphovascular invasion } \\
\hline Absent & 234 & $79(81.4)$ & $155(89.6)$ & 0.064 & $65(75.6)$ & $169(91.8)$ & $<0.001$ \\
\hline Present & 36 & $18(18.6)$ & $18(10.4)$ & & $21(24.4)$ & $15(8.2)$ & \\
\hline \multicolumn{8}{|c|}{ Perineural invasion } \\
\hline Absent & 226 & $76(78.4)$ & $150(86.7)$ & 0.087 & $64(74.4)$ & $162(88.0)$ & 0.007 \\
\hline Present & 44 & $21(21.6)$ & $23(13.3)$ & & $22(25.6)$ & $22(12.0)$ & \\
\hline \multicolumn{8}{|c|}{ Pathologic T category } \\
\hline pT1-2 & 220 & $78(80.4)$ & $142(82.1)$ & 0.746 & $64(74.4)$ & $156(84.8)$ & 0.045 \\
\hline pT3-4 & 50 & 19 (19.6) & $31(17.9)$ & & $22(25.6)$ & $28(15.2)$ & \\
\hline \multicolumn{8}{|c|}{ Lymph node metastasis } \\
\hline Absent & 149 & $50(51.5)$ & $99(57.2)$ & $>0.999$ & $35(40.7)$ & $114(62.0)$ & 0.002 \\
\hline Present & 121 & $47(48.5)$ & $74(42.8)$ & & $51(59.3)$ & $70(38.0)$ & \\
\hline \multicolumn{8}{|c|}{ Perinodal soft tissue extension ${ }^{\mathrm{b})}$} \\
\hline Absent & 30 & $12(37.5)$ & $18(37.5)$ & $>0.999$ & $11(32.4)$ & 19 (41.3) & 0.487 \\
\hline Present & 50 & $20(62.5)$ & $30(62.5)$ & & $23(67.6)$ & 27 (58.7) & \\
\hline
\end{tabular}

Values are presented as number (\%). HNSCC, head and neck squamous cell carcinoma; IHC, immunohistochemistry. ${ }^{\mathrm{a}}$ Trk immunohistochemistry was performed in all 275 cases but five cases could not be interpreted because of a damaged core, ${ }^{b}$ The perinodal soft tissue extension status was evaluated in 80 non-oropharyngeal HNSCC cases.

( $\mathrm{p}=0.045)$, and presence of lymph node metastasis $(\mathrm{p}=0.002)$ in non-oropharyngeal HNSCC (Table 3). Both TrkA and panTrk expression showed no significant correlation with perinodal soft tissue extension status. TrkB expression status showed no statistically significant correlation with any of the clinico-pathologic factors (S7 Table).

\section{Prognostic impact of Trk protein expression on HNSCC patients}

Among R0 resected HNSCCs ( $\mathrm{n}=305)$, five cases were not available for Kaplan-Meier analysis due to the absence of follow-up data. Among 300 cases of R0 resected HNSCCs, high TrkA expression showed a tendency to be related to superior OS (Fig. 4A), although it was not associated with RFS
(Fig. 4B). Especially in R0-resected oropharyngeal SCCs $(\mathrm{n}=82)$, high TrkA expression was significantly related to superior OS (Fig. 4C) and RFS (Fig. 4D). In non-oropharyngeal SCCs, especially in those of oral cavity $(n=181)$, high panTrk expression tended to be related to poor OS and inferior RFS than those with low panTrk expression (Fig. 4E and F). TrkB expression was not related to OS or RFS in oropharyngeal or non-oropharyngeal SCCs (S8 Fig.). Other nonoropharyngeal SCCs showed no significant correlation with the expression of TrkA and panTRK protein in Kaplan-Meier analysis (S9 and S10 Figs.).

In multivariate Cox analysis in $\mathrm{R} 0$ resected oropharyngeal HNSCC, only p16 expression status and pathologic T category acts as independent prognostic factors for OS and RFS (Table 4). However, in R0 resected oral cavity HNSCCs, age, 
A

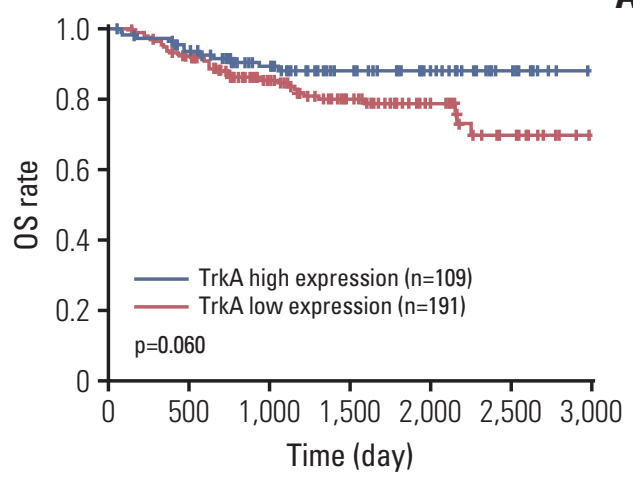

C

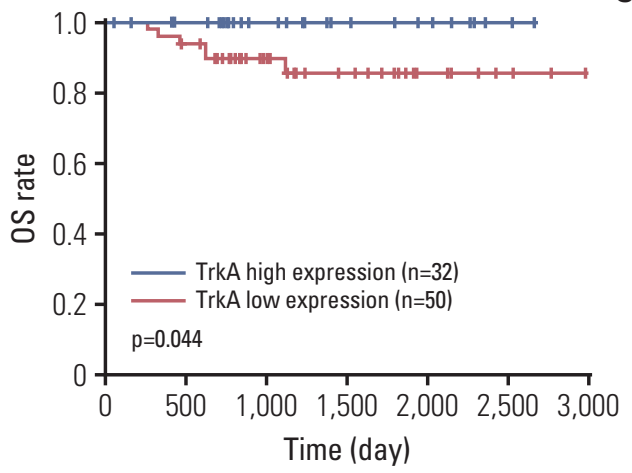

E

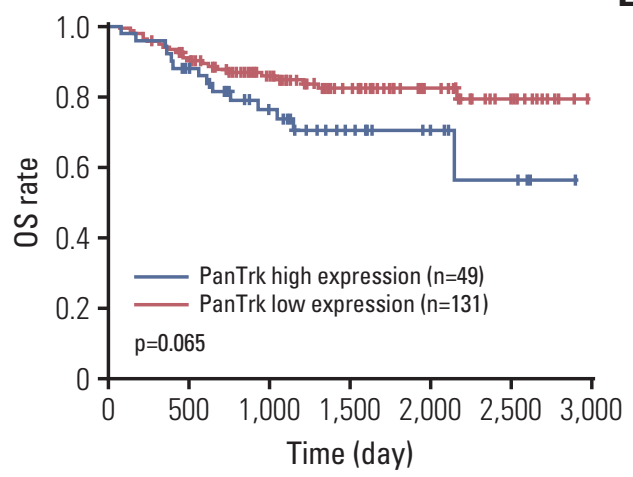

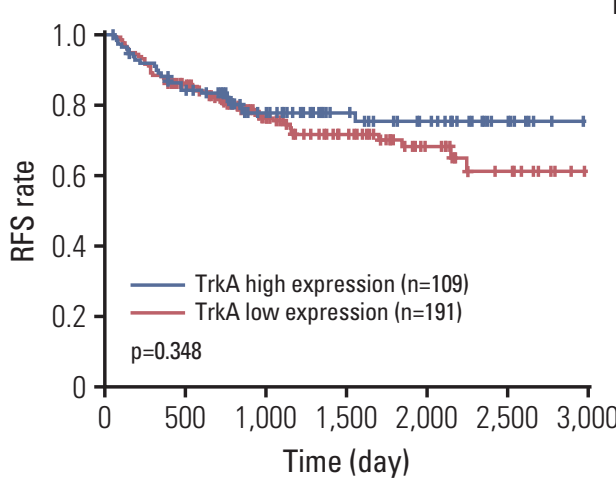

D

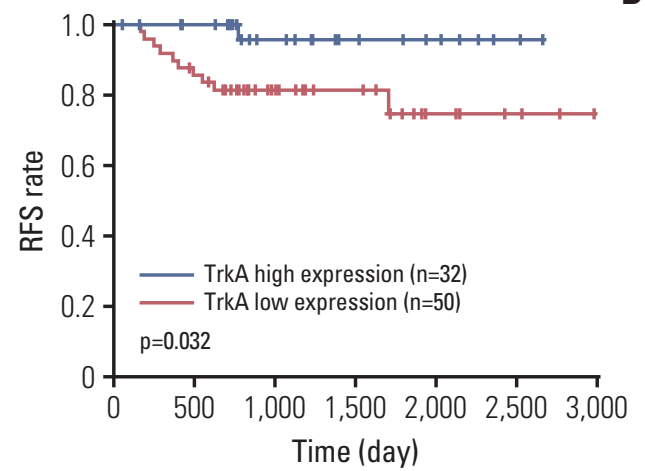

$\mathbf{F}$

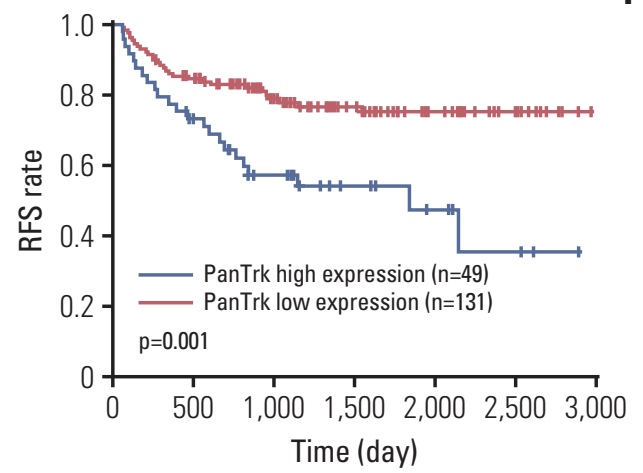

Fig. 4. Kaplan-Meier analysis of R0-resected head and neck squamous cell carcinomas (SCCs) in aspect of anatomical sites. (A) In the analysis of $300 \mathrm{R} 0$ resected cases, TrkA high expression group tended to have a longer overall survival (OS) than the Trk low expression group $(\mathrm{p}=0.060)$. (B) No significant difference was observed in recurrence-free survival (RFS) according to TrkA expression status ( $\mathrm{p}=0.348$ ). (C) In the analysis of $82 \mathrm{R} 0$ resected oropharyngeal SCCs, TrkA high group had a significantly longer OS than the TrkA low expression group ( $p=0.044)$. (D) In addition, the TrkA high group showed longer RFS than the TrkA low expression group ( $\mathrm{p}=0.032$ ). (E) In $180 \mathrm{R} 0$ resected oral cavity SCCs, high panTrk expression tended to be related to poor OS ( $\mathrm{p}=0.065)$. (F) Furthermore, the high panTrk group showed a significantly inferior RFS than the low panTrk group $(\mathrm{p}=0.001)$. 
Table 4. Univariate and multivariate analysis of RFS and OS in R0 resected oropharyngeal HNSCC ( $\mathrm{n}=82)$

\begin{tabular}{|c|c|c|c|c|c|c|c|c|}
\hline \multirow{3}{*}{ Variable } & \multicolumn{4}{|c|}{ OS } & \multicolumn{4}{|c|}{ RFS } \\
\hline & \multicolumn{2}{|c|}{ Univariate } & \multicolumn{2}{|c|}{ Multivariate } & \multicolumn{2}{|l|}{ Univariate } & \multicolumn{2}{|c|}{ Multivariate } \\
\hline & HR $(95 \%$ CI) & p-value & HR $(95 \%$ CI) & p-value & HR $(95 \% \mathrm{CI})$ & p-value & $\mathrm{HR}(95 \% \mathrm{CI})$ & p-value \\
\hline \multicolumn{9}{|l|}{ Age $\left.(y r)^{a}\right)$} \\
\hline$<58$ & 1 & & - & & 1 & & - & \\
\hline$\geq 58$ & $0.414(0.076-2.264)$ & 0.309 & - & - & $0.978(0.297-3.217)$ & 0.970 & - & - \\
\hline \multicolumn{9}{|l|}{ Sex } \\
\hline Female & 1 & & - & & 1 & & - & \\
\hline Male & $3.291(0.097-112.2)$ & 0.508 & - & - & $3.395(0.285-48.89)$ & 0.333 & - & - \\
\hline \multicolumn{9}{|l|}{ Smoking } \\
\hline Non-smoker & 1 & & - & & 1 & & - & \\
\hline Ex-smoker & $2.220(0.070-70.69)$ & 0.652 & - & - & $1.526(0.081-28.82)$ & 0.778 & - & - \\
\hline Smoker & $6.063(0.369-99.61)$ & 0.207 & - & - & $8.095(0.934-70.13)$ & 0.058 & - & - \\
\hline \multicolumn{9}{|l|}{$\begin{array}{l}\text { p16 IHC } \\
\text { status }\end{array}$} \\
\hline Negative & 1 & & 1 & & 1 & & 1 & \\
\hline Positive & $0.135(0.022-0.828)$ & 0.030 & $0.192(0.034-1.092)$ & 0.063 & $0.281(0.084-0.939)$ & 0.039 & $0.281(0.083-0.953)$ & 0.042 \\
\hline \multicolumn{9}{|c|}{$\begin{array}{l}\text { Lymphovascular } \\
\text { invasion }\end{array}$} \\
\hline Absent & 1 & & - & & 1 & & - & \\
\hline Present & $0.486(0.057-4.158)$ & 0.510 & - & - & $1.476(0.431-5.058)$ & 0.536 & - & - \\
\hline \multicolumn{9}{|l|}{$\begin{array}{l}\text { Perineural } \\
\text { invasion }\end{array}$} \\
\hline Absent & 1 & & - & & 1 & & - & \\
\hline Present & $0.346(0.001-101.0)$ & 0.714 & - & - & $0.345(0.005-22.01)$ & 0.616 & - & - \\
\hline \multicolumn{9}{|l|}{$\begin{array}{l}\text { Pathologic } \\
\text { T category }\end{array}$} \\
\hline pT1-2 & 1 & & 1 & & 1 & & 1 & \\
\hline pT3-4 & $11.65(2.327-58.34)$ & 0.003 & $11.305(2.173-58.803)$ & 0.004 & $7.038(2.048-24.19)$ & 0.002 & $7.041(2.023-24.51)$ & 0.002 \\
\hline \multicolumn{9}{|l|}{$\begin{array}{l}\text { Lymph node } \\
\text { metastasis }\end{array}$} \\
\hline Absent & 1 & & - & & 1 & & - & \\
\hline Present & $1.592(0.185-13.723)$ & 0.657 & - & - & $1.322(0.285-6.132)$ & 0.713 & - & - \\
\hline \multicolumn{9}{|l|}{$\begin{array}{l}\text { TrkA } \\
\text { expression }\end{array}$} \\
\hline Low & 1 & & - & & 1 & & - & \\
\hline High & $0.021(0.000-18.39)$ & 0.265 & - & - & $0.144(0.018-1.126)$ & 0.065 & - & - \\
\hline \multicolumn{9}{|l|}{$\begin{array}{l}\text { TrkB } \\
\text { expression }\end{array}$} \\
\hline Low & 1 & & - & & 1 & & - & \\
\hline High & $0.838(0.152-4.630)$ & 0.839 & - & - & $0.655(0.173-2.478)$ & 0.533 & - & - \\
\hline \multicolumn{9}{|l|}{$\begin{array}{l}\text { PanTrk } \\
\text { expression }\end{array}$} \\
\hline Low & 1 & & - & & 1 & & - & \\
\hline High & $0.416(0.084-2.060)$ & 0.282 & - & - & $0.466(0.142-1.531)$ & 0.208 & - & - \\
\hline
\end{tabular}

RFS, recurrence-free survival; OS, overall survival; HNSCC, head and neck squamous cell carcinoma; HR, hazard ratio; CI,

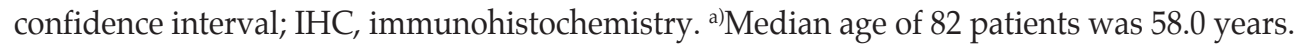


presence of lymphovascular invasion and advanced pathologic $\mathrm{T}$ category were independent factors for OS and RFS (S11 Table). Higher panTrk expression tends to relate with inferior RFS in multivariate analysis ( $\mathrm{p}=0.060)$ (S11 Table). In Kaplan-Meier analysis of 91 R1-resected head and neck squamous cell carcinomas in association with Trk expression, no significant difference was observed in OS and RFS according to TrkA, panTRkA, or TrkB expression (S12 Fig.).

\section{Discussion}

In this study, we evaluated and compared the expression status of subtypes of Trk in oropharyngeal cancer and nonoropharyngeal cancer and investigated the relationship between the expression levels of these subtypes and p16 expression status, clinicopathological variables, and patient prognosis.

In our study, cytoplasmic staining of tumors varied in intensity. Recent studies have described the reliability of panTrk and TrkA immunohistochemical staining in the prediction of NTRK gene alteration [23-25]: one study reported 15 cases of positive pan-Trk positive staining in 16 cases of known NTRK fusions. Interestingly, cytoplasmic staining accompanied by other expression patterns has been found to be associated with specific NTRK fusion patterns: EML4NTRK3 fusion with nuclear staining, LMNA-NTK1 fusion with nuclear membrane staining, and TPM3/4-NTRK1/3 and TRAF2-NTRK2 fusion with membranous staining [24]. Also, research suggest that strong cytoplasmic TrkA expression might be related with NTRK1 gene fusion in colorectal cancers [23] and might be indicative of gene copy number gain in solid tumors, such as lung adenocarcinoma, and colon and bile duct cancer [25].

We observed that TrkA and panTrk were expressed more highly in oropharyngeal than non-oropharyngeal SCCs. Oropharyngeal cancer shows a significant association with HPV infection, in contrast to non-oropharyngeal SCC. Carcinomas driven by HPV infection induce $\mathrm{p} 16$ protein overexpression through loss of control over cell cycle regulation; therefore, p16 immunohistochemistry is a reliable surrogate marker for HPV infection [18,19,21,22,26,27]. These previous findings and those of the present study suggest that high expression of TrkA and panTrk might be related to HPV-driven molecular changes during the carcinogenesis of oropharyngeal SCCs. In the present study, HPV gene transfection on the originally HPV-negative HNSCC cell-lines altered TrkA and TrkB gene expression. Especially, the increment of TrkA and TrkC gene expression after HPV gene transfection reflects our observation about the close correlation between high TrkA and panTrk $(\mathrm{A}+\mathrm{B}+\mathrm{C})$ expression and 16 positive HNSCC cases. From the findings, HPV infection may play a role in regulation of TrkA and TrkC expression. Further in-depth studies are needed for the detailed mechanisms related to the interaction between HPV infection and each Trk subtype regulation.

Besides HPV infection, genetic alteration may be related to alter Trk expression. According to recent The Cancer Genome Atlas data for HNSCCs (530 samples/528 patients; www. cbioportal.org/study?id=hnsc_tcga\#summary); however, mutations of the NTRK1, 2, and 3 genes have a very low frequency $(0.6 \%, 0.6 \%$, and $2.1 \%$, respectively), and copy number alterations (amplifications or deletions) are also low frequency $(1.2 \%, 0.8 \%$, and $0.6 \%$, respectively). These findings suggest that genetic mutations or copy number alterations are not the major factors underlying TrkA and panTrk overexpression in HNSCCs. A recent study of colorectal adenocarcinomas reported that high TrkA protein expression was related to NTRK1 gene rearrangement [28]. Although colorectal adenocarcinomas are very different from HNSCCs in terms of anatomical origin, histology, and biology of carcinogenesis, investigation of NTRK gene rearrangements should be performed to gain insights into the mechanism underlying TrkA and panTrk overexpression in HNSCCs.

In the present study, high TrkA expression was related to favorable prognosis. Given that HPV-positive oropharyngeal SCCs have a more favorable prognosis than HPV-negative carcinomas [29], and that high TrkA expression was associated with p16 positivity in the present study, close association between high TrkA expression and the favorable outcome of oropharyngeal SCCs may be related to HPV infection. Further in-depth study, however, may be necessary about the relations among HPV infection, Trk regulation, tumor cell growth.

In contrast, high panTrk expression was related to poor outcomes in patients with oral cavity SCCs, but couldn't prove as independent prognostic factor for OS and RFS. Regarding previous studies about pancreatic cancer and adenoid cystic carcinoma [30,31], overexpression of Trk protein is associated with tumor metastasis or perineural invasion which is concordant with our result. Given that panTrk includes TrkA, B, and C, among TrkA, B, and C or the combination of these Trk subtypes affects lymphovascular invasion and perineural invasion and contribute to the poor outcome in patients with oral cavity SCCs. However, we were not able to assess TrkC protein expression by immunohistochemistry, because of the lack of commercially available and reliable antibodies. Although further studies are required to identify the prognostic relevance of TrkC, oral cavity SCCs showing high panTrk expression may be candidates for treatment with panTrk inhibitors.

In previous in vitro study, TrkB expression may be related 
with tumor proliferation and chemotherapy resistance [32-34]. However, we could not prove the relationship between TrkB expression and OS or RFS. This might be limitation of immunohistochemical staining of TrkB protein. Since our study cohort is composed of surgically resected specimen without prior therapy, there may be need for TrkB expression status in samples of recurrent HNSCC or chemotherapy-resistant cases.

Trk protein expression seems to differ according to anatomical site and p16 expression or HPV status with clinical relevance. As a result, Trk expression status should be considered in the context of anatomical site and Trk subtype when considering treatment using Trk inhibitors.

\section{Electronic Supplementary Material}

Supplementary materials are available at Cancer Research and Treatment website (https:// www.e-crt.org).

\section{Conflicts of Interest}

Conflict of interest relevant to this article was not reported.

\section{Acknowledgments}

The study was supported by a grant from the Basic Science Research Program through the National Research Foundation of Korea (NRF), funded by the Ministry of Education (NRF-2015R1D1A1A09059399).

\section{References}

1. Chaturvedi AK, Anderson WF, Lortet-Tieulent J, Curado MP, Ferlay J, Franceschi S, et al. Worldwide trends in incidence rates for oral cavity and oropharyngeal cancers. J Clin Oncol. 2013;31:4550-9.

2. Sun S, Wang Z. Head neck squamous cell carcinoma c-Met(+) cells display cancer stem cell properties and are responsible for cisplatin-resistance and metastasis. Int J Cancer. 2011;129: 2337-48.

3. Hamilton D, Khan MK, O'Hara J, Paleri V. The changing landscape of oropharyngeal cancer management. J Laryngol Otol. 2017;131:3-7.

4. Westra WH, Lewis JS Jr. Update from the 4th Edition of the World Health Organization classification of head and neck tumours: oropharynx. Head Neck Pathol. 2017;11:41-7.

5. Westra WH, Boy S, El-Mofty SK, Gillison M, Schwartz MR, Syrjanen $\mathrm{S}$, et al. Squamous cell carcinoma, HPV-positive. In: El-Naggar AK, Chan JK, Grandis JR, Takata T, Slootweg PJ, editors. WHO classification of head and neck tumours. 4th ed. Lyon: IARC Press; 2017. p. 136-8.

6. Lewis JS Jr, Beadle B, Bishop JA, Chernock RD, Colasacco C, Lacchetti $C$, et al. Human papillomavirus testing in head and neck carcinomas: guideline from the College of American Pathologists. Arch Pathol Lab Med. 2018;142:559-97.

7. Fakhry C, Lacchetti C, Rooper LM, Jordan RC, Rischin D, Sturgis EM, et al. Human papillomavirus testing in head and neck carcinomas: ASCO clinical practice guideline endorsement of the College of American Pathologists guideline. J Clin Oncol. 2018;36:3152-61.

8. Thiele CJ, Li Z, McKee AE. On Trk--the TrkB signal transduction pathway is an increasingly important target in cancer biology. Clin Cancer Res. 2009;15:5962-7.

9. Kupferman ME, Jiffar T, El-Naggar A, Yilmaz T, Zhou G, Xie $\mathrm{T}$, et al. TrkB induces EMT and has a key role in invasion of head and neck squamous cell carcinoma. Oncogene. 2010;29: 2047-59.

10. Zhu L, Werner JA, Mandic R. Implications of tropomyosinrelated kinase $\mathrm{B}(\mathrm{TrkB})$ in head and neck cancer. Anticancer Res. 2007;27:3121-6.

11. Edge SB, Byrd DR, Compton CC, Fritz AG, Greene FL, Trotti A. AJCC cancer staging manual. 7th ed. New York: Springer; 2010.

12. Barnes L, Eveson JW, Reichart P, Sidransky D. World Health Organization classfication of tumours: pathology and genetics of head and neck tumours. Lyon: IARC Press; 2005.

13. Cho YA, Kim EK, Heo SJ, Cho BC, Kim HR, Chung JM, et al. Alteration status and prognostic value of MET in head and neck squamous cell carcinoma. J Cancer. 2016;7:2197-206.

14. Yun S, Koh JM, Lee KS, Seo AN, Nam KH, Choe G. Expression of c-MET in invasive meningioma. J Pathol Transl Med. 2015;49:44-51.

15. Shin JH, Kim CJ, Jeon EJ, Sung CO, Shin HJ, Choi J, et al. Overexpression of $\mathrm{C}$-reactive protein as a poor prognostic marker of resectable hepatocellular carcinomas. J Pathol Transl Med. 2015;49:105-11.

16. Park E, Park SY, Kim H, Sun PL, Jin Y, Cho SK, et al. Membranous insulin-like growth factor-1 receptor (IGF1R) expression is predictive of poor prognosis in patients with epidermal growth factor receptor (EGFR)-mutant lung adenocarcinoma. J Pathol Transl Med. 2015;49:382-8.

17. Bishop JA, Ma XJ, Wang H, Luo Y, Illei PB, Begum S, et al. Detection of transcriptionally active high-risk HPV in patients with head and neck squamous cell carcinoma as visualized by a novel E6/E7 mRNA in situ hybridization method. Am J Surg Pathol. 2012;36:1874-82.

18. Mirghani H, Casiraghi O, Amen F, He M, Ma XJ, Saulnier P, et al. Diagnosis of HPV-driven head and neck cancer with a 
single test in routine clinical practice. Mod Pathol. 2015;28: 1518-27.

19. Ang KK, Harris J, Wheeler R, Weber R, Rosenthal DI, NguyenTan PF, et al. Human papillomavirus and survival of patients with oropharyngeal cancer. N Engl J Med. 2010;363:24-35.

20. Lewis JS Jr, Chernock RD, Ma XJ, Flanagan JJ, Luo Y, Gao G, et al. Partial p16 staining in oropharyngeal squamous cell carcinoma: extent and pattern correlate with human papillomavirus RNA status. Mod Pathol. 2012;25:1212-20.

21. Castellsague X, Alemany L, Quer M, Halec G, Quiros B, Tous $\mathrm{S}$, et al. HPV involvement in head and neck cancers: comprehensive assessment of biomarkers in 3680 patients. J Natl Cancer Inst. 2016;108:djv403.

22. Lewis JS Jr. p16 immunohistochemistry as a standalone test for risk stratification in oropharyngeal squamous cell carcinoma. Head Neck Pathol. 2012;6 Suppl 1:S75-82.

23. Choi Y, Won YJ, Lee S, Kim A, Kim Y, Park WY, et al. Cytoplasmic TrkA expression as a screen for detecting NTRK1 fusions in colorectal cancer. Transl Oncol. 2018;11:764-70.

24. Hechtman JF, Benayed R, Hyman DM, Drilon A, Zehir A, Frosina D, et al. Pan-Trk immunohistochemistry is an efficient and reliable screen for the detection of NTRK fusions. Am J Surg Pathol. 2017;41:1547-51.

25. Mauri G, Valtorta E, Cerea G, Amatu A, Schirru M, Marrapese $\mathrm{G}$, et al. TRKA expression and NTRK1 gene copy number across solid tumours. J Clin Pathol. 2018;71:926-31.

26. Ndiaye C, Mena M, Alemany L, Arbyn M, Castellsague X, Laporte L, et al. HPV DNA, E6/E7 mRNA, and p16INK4a detection in head and neck cancers: a systematic review and meta-analysis. Lancet Oncol. 2014;15:1319-31.
27. Nevens D, Nuyts S. HPV-positive head and neck tumours, a distinct clinical entity. B-ENT. 2015;11:81-7.

28. Lee SJ, Li GG, Kim ST, Hong ME, Jang J, Yoon N, et al. NTRK1 rearrangement in colorectal cancer patients: evidence for actionable target using patient-derived tumor cell line. Oncotarget. 2015;6:39028-35.

29. Feller L, Wood NH, Khammissa RA, Lemmer J. Human papillomavirus-mediated carcinogenesis and HPV-associated oral and oropharyngeal squamous cell carcinoma. Part 2: Human papillomavirus associated oral and oropharyngeal squamous cell carcinoma. Head Face Med. 2010;6:15.

30. Shan C, Wei J, Hou R, Wu B, Yang Z, Wang L, et al. Schwann cells promote EMT and the Schwann-like differentiation of salivary adenoid cystic carcinoma cells via the BDNF/TrkB axis. Oncol Rep. 2016;35:427-35.

31. Sclabas GM, Fujioka S, Schmidt C, Li Z, Frederick WA, Yang $\mathrm{W}$, et al. Overexpression of tropomysin-related kinase B in metastatic human pancreatic cancer cells. Clin Cancer Res. 2005;11(2 Pt 1):440-9.

32. Lee J, Jiffar T, Kupferman ME. A novel role for BDNF-TrkB in the regulation of chemotherapy resistance in head and neck squamous cell carcinoma. PLoS One. 2012;7:e30246.

33. Yilmaz T, Jiffar T, de la Garza G, Lin H, Milas Z, Takahashi Y, et al. Theraputic targeting of Trk supresses tumor proliferation and enhances cisplatin activity in HNSCC. Cancer Biol Ther. 2010;10:644-53.

34. Pearse RN, Swendeman SL, Li Y, Rafii D, Hempstead BL. A neurotrophin axis in myeloma: TrkB and BDNF promote tumor-cell survival. Blood. 2005;105:4429-36. 